\title{
Toward the Solution of the Inverse Problem in Neutron Reflectometry
}

\author{
V. O. De Haan and A. A. Van Well \\ I.R.I., Delft University \\ 2629 JB Delft, The Netherlands \\ Paul E. Sacks \\ Department of Mathematics \\ Iowa State University \\ Ames, IA 50010 \\ and
S. Adenwalla and G. P. Felcher
Argonne National Laboratory
Argonne, Il 60439

RECEIVED

DIIr 041997

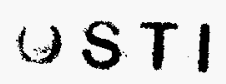

87579

\section{ANL/MSD/PP--87579}

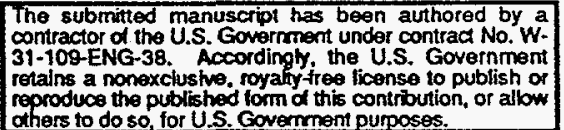

August, 1995

Submitted to Physica B

\section{DISCLAIMER}

/sm

Distribution:

1-2. M. J. Masek

3. B. D. Dunlap

4. G. W. Crabtree

5. J. D. Jorgensen

6. B. S. Brown

7. Editorial Office

8. Authors

This report was prepared as an account of work sponsored by an agency of the United States Government. Neither the United States Government nor any agency thereof, nor any of their employees, makes any warranty, express or implied, or assumes any legal liability or responsibility for the accuracy, completeness, or usefulness of any information, apparatus, product, or process disclosed, or represents that its use would not infringe privately owned rights. Reference herein to any specific commercial product, process, or service by trade name, trademark, manufacturer, or otherwise does not necessarily constitute or imply its endorsement, recommendation, or favoring by the United States Government or any agency thereof. The views and opinions of authors expressed herein do not necessarily state or reflect those of the United States Government or any agency thereof.

Uniled Stares Government or any agency thereor.

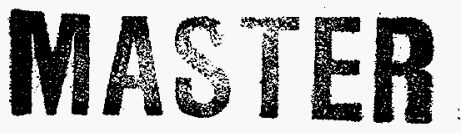

DISTPIBUTION OF THIS DOCUMENT IS UNLIMTED

This work is supported by the Division of Materials Sciences, Office of Basic Energy Sclences of DOE, under contract No. W-31-109-ENG-38. 


\section{DISCLAMMER}

Portions of this docoment may be illegible in electronic image products. Images are produced from the best available original document. 


\title{
TOWARD THE SOLLTION OF THE INVERSE PROBLEM \\ IN NEUTRON REFLECTOMETRY
}

\author{
V.O. de Haan and A.A. van Well \\ Interfacultair Reactor Instituut. Delft University of Technology, 2629 JB Delft. \\ The Netherlands \\ P.E. Sacks \\ Dept. of Mathematics, Iowa State University, Ames, Iowa 50011, USA \\ and \\ S. Adenwalla and G.P. Felcher \\ Argonne National Laboratory, Argonne, Illinois 60439, USA
}

\section{abstract}

We show that the chemical depth profile of a film of unknown structure can be retrieved unambiguously from neutron reflection data by adding to the system a known magnetic layer. Three independent reflectivities are obtained by taking measurements with the sample magnetized in a magnetic field perpendicular to the surface and subsequently parallel to it, and using in the latter geometry neutrons polarized either in the direction of the field or opposite to it. The procedure consists of two steps. First, from the three reflectivities both the real and imaginary parts of the reflection coefficient of the unknown film are extracted within the framework of the rigorous dynamical theory. Second. the neutron scattering-length density (and consequently the chemical depth profile) is obtained by a suitable numerical technique for the conventional Schrödinger inverse scattering problem. Computer experiments were conducted for selected cases: starting from the profiles the reflectivities ware calculated in a limited range of $q$ and then the original profiles were successfully recovered. The influence on the accuracy of the recovered depth profile of the counting statistics and the cutoffs at low and high $q$ are discussed.

The submitted manuscript has been authored by a contractor of the U.S. Government under contract No. W-31-109ENG-38. Accordingly, the U.S. Government retains o nonexclusive, royalty-free license to publist or reproduce the publistied form of this contribution, or allow others to do so, for 


\section{Introduction}

Neutron reflection experiments are important in understanding the physics of many surface and interfacial structures, in fields as diverse as polymers and magnetism [1-2]. The measurement of the reflected intensity (hereafter referred to as reflectivity) $R(q)$ as a function of the perpendicular component of the incoming wave vector $q=2 \pi \sin$ $\theta / \lambda$, with $\lambda$ the neutron wavelength and $\theta$ the reflection angle, provides information about the atomic or magnetic density profile of the sample along its depth $z$. The reflectivity is the square of the complex reflection coefficient $r(q)$. For a given scattering-length density profile $\Gamma(z)$, the reflection coefficient can be calculated straightforwardly, e.g. by means of the optical matrix method [3]

$$
\Gamma(z)=4 \pi N(z)(b(z) \pm p(z)) \leftrightarrow r(q)
$$

Here, $N(z)$ is the atomic number density and $b(z)$ the average coherent scattering length. If the reflecting sample is magnetized parallel to the interface, also the average 'magnetic scattering length', $p(z)=C \mu$, contributes to $\Gamma(z)$, where $\mu$ is the average magnetic moment per atom in units Bohr magneton $\mu_{B}$, and $C=2.70 \mathrm{fm} / \mu_{\mathrm{B}}$. The + and - signs represent spin-up (neutron spin parallel to magnetization) and spin-down states, respectively. If $r(q)$ were known in amplitude and phase, the inversion of Eq.(1) can in principle be performed, and practical algorithms have been developed [4-9]. If the phase is not known, as is the case in a standard reflection experiment where only $R(q)$ is determined, generally least-squares methods are used to determine $\Gamma(z)$ [10-11], but in general the solution is not unique. In the literature, methods to retrieve phase information from the measured reflectivity are discussed [12-16]. For one reflectivity profile these methods yield many solutions for the phase. stressing the non-uniqueness of the solution. To decide which solution is correct, extra information is required. This information could be knowledge of the structure as deduced from either its preparation or its analysis by way of complementary depth-profiling techniques [17]. Another way to get more information about the sample is the use of contrast variation in neutron reflectivity as obtained for instance by isotopic substitution [18-19].

Phase information can be obtained by depositing the unknown (non-magnetic) film on a magnetized reference substrate, and carrying out the measurements with polarized neutrons. Sivia and Pynn [20] and Majkrzak et al. [21] have shown schemes for 
inverting the spin-up and spin-down reflectivity data to obtain the chemical density profile of the unknown layer. However, their method is based on the first Born approximation (kinematic limit) and is only valid for $q$ values considerably larger than the critical value. For reflection data in the dynamical regime (smaller $q$ values, larger reflectivities), Majkrzak et al. [21] applied an analogous scheme within the distorted wave Born approximation, but this did not lead to unambiguous results. De Haan et al. [22] discussed a method to retrieve the amplitude and phase of the reflection coefficient exactly, even in the dynamical regime. Independently, Majkrzak and Berk [23] proposed a method that is essentially identical. In this method a known and tunable reference layer is added to the unknown layer. From the reflectivities, measured with three values of the reference layer, the complex reflection coefficient $r(q)$ of the unknown layer can be calculated unambiguously, for each $q$ value. Here we will discuss the method and apply an inversion scheme to recover the scattering-length density. To demonstrate the practical use of the method, we carried out 'computer experiments' and examined the influence of statistical uncertainties in the experimental data.

The lay out of the paper is as follows. In Section 2 we introduce some examples of the behaviour of $r(q)$ in the complex plane. We show that two different $\Gamma(z)$ profiles can give the same amplitude $|r(q)|$. The method to retrieve the phase information ('three circle method') is described in Section 3, while in Section 4 the inversion of the recovered $r(q)$ data is discussed. The three circle method and the inversion scheme are applied to the examples introduced in Section 2. The recovered $\Gamma(z)$ s are compared with the start values, in the case of 'perfect' experiments. The influence of experimental uncertainty on both the three circle method and the inversion are investigated, by adding $5 \%$ Gaussian scatter to the exact $R(q)$ values. In Section 5 we end with some concluding remarks.

\section{Reflection coefficient for one layer: an example}

The reflection and transmission coefficients of a complex system are determined by

$$
\left(\begin{array}{l}
t \\
0
\end{array}\right)=\mathrm{M}\left(\begin{array}{l}
1 \\
r
\end{array}\right)=\left(\begin{array}{ll}
m_{11} & m_{12} \\
m_{21} & m_{22}
\end{array}\right)\left(\begin{array}{l}
1 \\
r
\end{array}\right) .
$$


For a system that may be decomposed in a histogram of $n$ layers, $M=M_{n} M_{n-1} \ldots \ldots M_{1} M_{0}$. with

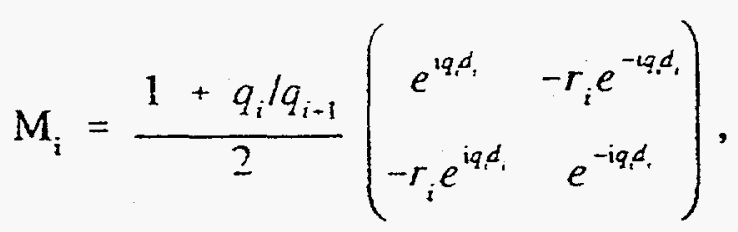

where $q_{\mathrm{i}}=\left(q_{\mathrm{vacum}}{ }^{2}-\Gamma_{\mathrm{i}}\right)^{1 / 2}$ is the vertical component of the wave vector in, and $d_{\mathrm{i}}$ the thickness of layer $i$. The reflection coefficient $r_{i}$ represents the reflection of the interface between $i$ and $i+1$, and is given by $r_{i}=D_{i} r_{i}^{F}$, where $r_{i}^{F}$ is the Fresnel reflection coefficient

$$
r_{i}^{F}=\frac{q_{i}-q_{i+1}}{q_{i}+q_{i+1}}
$$

and $D_{\mathrm{i}}$ describes the interfacial roughness

$$
D_{i}=\mathrm{e}^{-2 q_{q_{i-2}} \sigma_{0},},
$$

with $\sigma_{\mathrm{i}}$ the rms roughness parameter of the $\mathrm{i} / \mathrm{i}+1$ interface. For a single layer, the reflection coefficient is

$$
r(q)=\frac{r_{0}+r_{1} \mathrm{e}^{2 \mathrm{i}_{1} d_{1}}}{1+r_{0} r_{1} \mathrm{e}^{2 \mathrm{i}_{1} d_{1}}} .
$$

If we consider a substrate with $\Gamma=0$ (i.e. $q_{0}=q_{2}=q$ ), then $r_{0}{ }^{\mathrm{F}}=-r_{1}^{\mathrm{F}}$, and for large $q$ values Eq. (6) approaches

$$
r(q)=\frac{\Gamma_{1}}{4 q^{2}}\left(D_{0}-D_{1} \mathrm{e}^{2 \mathrm{i} q_{1} d_{1}}\right)
$$

In Figs. 1-3 examples are shown for a $25 \mathrm{~nm}$ thick gold layer, with $\Gamma_{1}=0.00554 \mathrm{~nm}^{-2}$. The result of introducing a roughness of 2.5 on the first and/or the second interface is demonstrated. In Fig. 3, the large $q$ behaviour, given in Eq. (7), is evident. The amplitudes $|r(q)|$ for Au01 and Au10 are identical. The phases of $r(q)$ for Au00 and Aull are on the scale of the plot indistinguishable. The maximum discrepancy of approximately $2 \%$ arises for $q \approx 0.07 \mathrm{~nm}^{-1}$. This example demonstrates once more that for an unambiguous interpretation, both amplitude and phase are needed. 
3. Retrieval of phase information

Assume a film on top of the substrate consisting of two parts, whose reflection properties are described by the matrices $G$ and $H$. The neutron beam enters through the substrate and pass first through $H$, then $G$. Part $G$ represents the 'unknown' layer and part $\mathrm{H}$ is the tunable reference layer. For a given $q$, the reflection and transmission coefficient $r$ and $t$ are determined by the matrix relation

$$
\left(\begin{array}{l}
t \\
0
\end{array}\right)=\mathrm{M}\left(\begin{array}{l}
1 \\
r
\end{array}\right)=\mathrm{GH}\left(\begin{array}{l}
1 \\
r
\end{array}\right) .
$$

Then the reflection coefficient of the total system is given by

$$
r=-\frac{m_{21}}{m_{22}}=-\frac{h_{11} r_{g}-h_{21}}{h_{12} r_{g}-h_{22}},
$$

where $r_{g}=-g_{21} / g_{22}$ is the reflection coefficient of the unknown layer. Using Eq. (9), $r_{g}$ can be expressed in $R=r^{*}$ and the known elements of matrix $H$, i.e. $r_{g}$ is a point on a circle in the complex plane with centre $\gamma$ and radius $\rho$, given by

$$
\gamma=\frac{R h_{22} h_{12}^{*}-h_{21} h_{11}^{*}}{R h_{12} h_{12}^{*}-h_{11} h_{11}^{*}}
$$

and

$$
\rho=\sqrt{R} \frac{\text { Det } H}{R h_{12} h_{12}^{*}-h_{11} h_{11}^{*}},
$$

with Det $\mathrm{H}=\left|h_{11} h_{22}-h_{12} h_{21}\right|$. Both amplitude and phase of $r_{\mathrm{g}}$ can be uniquely determined, for each $q$ value independently, from three measurements of $R$ for three different values of $\mathrm{H}$ as the intersection of the three corresponding circles. In the Appendix the expression for the intersection point is given.

To facilitate the decomposition of matrix $M$ into the matrices $G$ and $H$, an extra layer $j$ with zero thickness and arbitrary $\Gamma_{j}$ is added inbetween layers $i$ and $i+1$. This means that for the matrix calculation of the known system the 'substrate' $-\Gamma$ must be taken $\Gamma_{j}$. 
Then. for the recovered profile of the unknown system $\Gamma(z)=\Gamma$, for $z<0$. If the interface between layer $i$ and $i+1$ is sharp, $\sigma_{i}=0$, it can be proven that the matrices $M$ for the systems with and without layer $\mathrm{j}$ are identical, i.e. $\mathrm{M}_{\mathrm{i}}=\mathrm{M}_{j} \mathrm{M}_{\mathrm{i}}^{\prime}$, where $\mathrm{M}_{\mathrm{i}}^{\prime}$ and $\mathrm{M}_{\mathrm{j}}$ describe the interfaces $\mathrm{i} / \mathrm{j}$ and $\mathrm{j} / \mathrm{i}+1$, respectively, and $d_{\mathrm{j}}=0$. If the interface $\mathrm{i} / \mathrm{i}+1$ is rough, a decomposition $M=G H$ is only possible with $\Gamma_{j}=\Gamma_{j}$, the roughnesses of the $\mathrm{i} / \mathrm{i}+1$ and $\mathrm{j} / \mathrm{i}+1$ interfaces equal, and a sharp $\mathrm{i} / \mathrm{j}$ interface.

To demonstrate the three circle method, we consider the systems sketched in Fig. 4. The scattering-length density of the substrate (incident medium) is taken $\Gamma=0$. The reference layer consists of a $25 \mathrm{~nm}$ thick cobalt film plus a $50 \mathrm{~nm}$ thick gap (or film with $\Gamma=0$ ). The scattering-length density is tuned by altering the magnetization of the cobalt layer. The unknown layer is a $25 \mathrm{~nm}$ thick gold layer with a sharp first and rough second interface (denoted Au01) and vice-versa (Au10). The roughness on both Co interfaces is $0.5 \mathrm{~nm}$. For both systems, three computer-simulated reflectivities have been calculated. Two mimicked experiments with Co magnetized parallel to the surface, and neutrons polarized parallel $(+)$ or anti-parallel $(-)$ to the Co magnetization. and a third with unpolarized neutrons and Co magnetized perpendicular to the surface (1). The corresponding scattering-length density profiles are given in Fig. 4(b). The three reflectivities $R$ were calculated exactly, and the intersection of the three corresponding circles was calculated, using the expression given in the Appendix. In Figs. 5(b-c) and $6(b-c)$ both the amplitude and phase of the recovered reflection coefficients are shown as a function of $q$. The reflection coefficients, recovered from the 'perfect data' coincide with the theoretical values. In order to simulate a real experiment, $5 \%$ statistics (Gaussian distribution with standard deviation $\sigma_{\mathrm{R}} / R=0.05$ ) was added to the three simulated reflectivities (Figs. 5(a) and 6(a)). Again, to calculate the intersections of circles, the expression in the Appendix is used. In Fig. (7) a few examples are given. For the noisy data at small $q$ values (the quasi total reflection region $\left.q<\Gamma_{\mathrm{Au}}^{4 / 2}\right)$, it is not possible to determine the intersection of the three circles accurately. In some cases two of the circles do not even intersect. Then the expression used to calculate the intersection point may give unphysical values with $|r(q)|>1$. The uncertainty in the resulting reflection coefficient is calculated by using the method of propagation of errors. 


\section{Inversion}

To reconstruct the scattering-length density (or potential) from the complex reflection coefficient, we use the method already described in the literature [8]. It gives accurate and unique $\Gamma(z)$ results if the following conditions are met: $\bullet \Gamma(z) \equiv 0$ for $z<z_{0} ; \bullet \Gamma(z)$ $-\Gamma(\infty)$ decays to zero sufficiently rapidly for large $z$; The real or the imaginary of $r(q)$ is accurately known for $0<q<\infty ; \bullet \Gamma(z)$ is real; $\bullet$ There are no bound states; this is the case if $\Gamma(z) \geq 0$. The numerical method applied does not use the standard GelfandLevitan-Marchenko (GLM) integral equation [24], but instead makes use of a fast and accurate layer stripping method, which may be employed after a transformation is made to an equivalent 'time domain' inverse problem involving the determination of . an unknown coefficient in a wave equation. See [8] for more details, including a discussion of some advantages of this method over the GLM-method.

The method was applied to the two examples given in Section 3. For the system Au01 $z_{0}=0$ was chosen, for system Au10 $z_{0}=-10 \mathrm{~nm}$. With perfect data, without band limitation, i.e. $r(q)$ is known for all $q>0, \Gamma(z)$ is reconstructed correctly. In our example, with $r(q)$ given for 250 equidistant $q$ values in the range $0<q \leq 0.5$, the reconstructed profile is represented by open circles in Fig. 8. The oscillations in Au10 (Fig. 8(b)) are due to band limitation. These oscillations are not present on Au01 (Fig.8(a)) because the program used automatically extrapolates the large $q$ behaviour in the case that the potential has a sharp interface at $z=z_{0}$ and no other sharp interface. As a next step, the noisy data (shown in Figs. $5(b-c)$ and $6(b-c)$ ) are used as input for the reconstruction program. The result is complete nonsense (triangles in Fig. 8). This is because the small- $q$ behaviour is very significant for the program, while the phase retrieval algorithm fails for noisy data at the smaller $q$ values. Fortunately, the value of $r(q)$ at $q=0$ is known. i.e. $r(0)=-1$, or $\operatorname{|r}(0) \mid=1$ and $\arg (r(0))=-\pi$. So, extrapolation to $q=0$ seems to be practically possible. To demonstrate the capability of the reconstruction method if the small- $q$ behaviour were known, we replace the $r(q)$ values of the noisy data by the perfect data for $q<\Gamma_{\mathrm{Au}}{ }^{2 / 2}$. Results are shown in Fig. 8 . It appears that the original $\Gamma$ profiles are recovered reasonably well. 


\section{Discussion}

We showed that it is possible to re:over the scattering-length density profile of a nonmagnetic layer within the dynamical theory, by performing three measurements with a magnetic layer as a tunable reference layer. With the three circle method is determined the complex reflection coefficient (i.e. both amplitude and phase) of the unknown layer. Using the inversion algorithm of [8], the scattering-length density profile is reconstructed uniquely. We confirmed the feasibility of the method by a computer experiment. In contrast with a physical experiment, a computer experiment is a real test, because here the density depth profile to be recovered is exactly. known. In our computer experiment we used reflectivity data down to $R=10^{-5}$, with a statistical accuracy of $5 \%$. These conditions are well exceeded in state-of-the-art neutron reflection facilities. The oaly point where our simulations up to now do not approach real experimental conditions is the negligence of the instrumental $q$ resolution.

It might be argued that the examples we chose were too particular to represent a sweeping experimental confrontation of the method. Here we would like to adduce the reasons for our choice and to discuss the path that we are undertaking to make our treatment more general. The basic features of our compound system (reference + sample) was free suspended, the front and back materials having the same scatteringlength density, and actually the reference was separated from the sample by a gap. The reference/sample gap, which made a system reminiscent of the geometry introduced in speckle holography [25] was really not necessary, and was introduced here simply because we wanted to explore the difference in the reflection coefficient for the samples rough in the front or in the back face. It would be nice if we could physically separate the 'known' from the 'unknown' system. E.g. as known system an 'air' substrate with a magnetic top layer, and as unknown system the unknown layer on an 'air' substrate. The measurements should be performed with an air gap of say $50 \mathrm{~nm}$ between the magnetic and the unknown layer. In practice, it will be difficult to place the two systems parallel (with a few $\mathrm{nm}$ accuracy) over an area of several $\mathrm{cm}^{2}$. Experimentally more feasible seems to be a reference system consisting of a silicon block, coated with a suitable magnetic layer. This system can be characterized separately. On top of it the unknown layer can be added. The neutron beam should 
enter the silicon from the side. and intemally reflect from the unknown layer. However, because $\Gamma_{S i}>0$, the inversion is more complicated.

The potentials, or scattering-length densities, $\Gamma(z)$ considered in this article have all been assumed to decay to zero sufficiently rapidly as $z \rightarrow \pm \infty$, but for more realistic application to neutron or $\mathrm{x}$-ray reflectometry, it is necessary to allow potentials having distinct limits at $\pm \infty$. The mathematical theory of inverse scattering in this case has been developed, e.g. in [26-27]. Assuming as before that the neutrons are incident from $-\infty$. one must consider separately the two cases $\Gamma(+\infty)>\Gamma(-\infty)$ and $\Gamma(+\infty)<\Gamma(-\infty)$. In either case one assumes that the reflectivity $R=r r^{*}$ may be measured for $q^{2}>\Gamma(-\infty)$. The first case, $\Gamma(+\infty)>\Gamma(-\infty)$, is somewhat simpler because knowledge of $r$ for all $q$ in principle suffices to determine the potential uniquely. The three circle method may still be adapted to recover the phase information, but only for $q^{2}>\Gamma(+\infty)$. The reason for this is that $R(q)=1$ whenever $\Gamma(-\infty)<q^{2}<\Gamma(+\infty)$, thus there can be no information in this data. The three circles must coincide in this range. We are working on a method for filling in the missing small- $q$ phase data. If this can be accomplished, the numerical method of [8] may again be used to compute the scattering-length density. The remaining case when $\Gamma(+\infty)<\Gamma(-\infty)$ will need a different idea, because even if phase information is available, the complex reflection coefficient $r(q)$ for $q^{2}>$ $\Gamma(-\infty)$ is not in principle adequate to uniquely determine the potential. According to [26] or [27], unique recovery is possible if the complex reflection coefficient is supplemented by extra data. as it may be obtained by looking at the system with neutrons incident from $+\infty$.

The methods that we are proposing, although seemingly bulky and complicated at the present stage of development. may find practical application in a type of experiments which is requiring an increasing role. This is when it is desired to measure the near surface composition at the boundary of a phase diagram, obtained over a range of an external variable (temperature, pressure, etc.) A spot check with the methods outlined here confirms the uniqueness of the phaseless solution; and confirms it with a resolution unmatched by other complementary depth-profiling techniques. 
Acknowledgements

Work at Argonne was supported by US-DOE, BES-MS contract the work of P.E. Sacks was supported in part by the National Science Foundation under contract DMS-9504611.

Appendix. Intersection of three circles

The three circles in the complex plane (Section 3) with centres $\gamma_{i}$ and radii $\rho_{i}$, corresponding with the three measurements, are given by

$$
\left|r-\gamma_{i}\right|^{2}=\rho_{i}^{2}
$$

The line, connecting the two intersection points of two circles, denoted by $i$ and $j$, is . represented by

$$
r^{*}\left(\gamma_{i}-\gamma_{j}\right)+r\left(\gamma_{i}^{*}-\gamma_{j}^{*}\right)=A_{i}-A_{j}
$$

with

$$
A_{i}=\gamma_{i} \gamma_{i}^{*}-\rho_{i}^{2}
$$

The three intersecting lines coincide in one point $r$

$$
r=\frac{A_{1}\left(\gamma_{2}-\gamma_{3}\right)+A_{2}\left(\gamma_{3}-\gamma_{1}\right)+A_{3}\left(\gamma_{1}-\gamma_{2}\right)}{\gamma_{1}^{*}\left(\gamma_{2}-\gamma_{3}\right)+\gamma_{2}^{*}\left(\gamma_{3}-\gamma_{1}\right)+\gamma_{3}^{*}\left(\gamma_{1}-\gamma_{2}\right)}
$$

If we define

$$
r=x+i y, \quad \gamma_{i}=\alpha_{i}+i \beta_{i},
$$

then $A_{i}=\alpha_{i}^{2}+\beta_{i}^{2}-\rho_{i}^{2}$, and the line of Eq. (15) reads

$$
2 x\left(\alpha_{i}-\alpha_{j}\right)+2 y\left(\beta_{i}-\beta_{j}\right)=A_{i}-A_{j}
$$


The real and imaginary part of the calculated reflection coefficient $r$ (Eq. (15)) can be expressed as

$$
x=\frac{1}{2} \frac{A_{1}\left(\beta_{2}-\beta_{3}\right)+A_{2}\left(\beta_{3}-\beta_{1}\right)+A_{3}\left(\beta_{1}-\beta_{2}\right)}{\alpha_{1}\left(\beta_{2}-\beta_{3}\right)+\alpha_{2}\left(\beta_{3}-\beta_{1}\right)+\alpha_{3}\left(\beta_{1}-\beta_{2}\right)},
$$

and

$$
y=\frac{1}{2} \frac{A_{1}\left(\alpha_{2}-\alpha_{3}\right)+A_{2}\left(\alpha_{3}-\alpha_{1}\right)+A_{3}\left(\alpha_{1}-\alpha_{2}\right)}{\beta_{1}\left(\alpha_{2}-\alpha_{3}\right)+\beta_{2}\left(\alpha_{3}-\alpha_{1}\right)+\beta_{3}\left(\alpha_{1}-\alpha_{2}\right)} .
$$

\section{References}

[1] H. Zabel, I.K. Robinson, (Eds.), 'Surface X-Ray and Neutron Scattering', (Springer Verlag, Berlin, 1992).

[2] H.J. Lauter, V.V. Pasyuk, (Eds.), 'Proceedings of the International Conference on Surface X-Ray and Neutron Scattering SXNS3', Physica B 198 (1994) 1266.

[3] J. Lekner, 'Theory of Reflection', Martinus Nijhof publishers, Dordrecht, 1987).

[4] H.E. Moses, Phys. Rev. 102 (1956) 559.

[5] R.G. Newton, SIAM Rev. 12 (1970) 346.

[6] B.N. Zakhariev, A.A. Suzko. 'Direct and Inverse Problems', (Springer Verlag, Heidelberg, 1990).

[7] T.M. Roberts, Physica B 173 (1991) 143.

[8] P.E. Sacks, Wave Motion 18 (1993) 21.

[9] N.F. Berk and C.F. Majkrzak, this conference.

[10] V.O. de Haan and G.G. Drijkoningen, Physica B 198 (1994) 24.

[11] X.L. Zhou and S.H. Chen, Phys. Rev. E 147 (1993) 121.

[12] R.E. Burge, M.A. Fiddy, A.H. Greenaway, and G. Ross, Proc. R. Soc. Lodon. A 350 (1976) 191.

[13] M.V. Klibanov and P.E. Sacks. J. Math. Phys. 33 (1992) 3813.

[14] M.V. Klibanov and P.E. Sacks, J. Comput. Phys. 112 (1994) 273.

[15] M.V. Klibanov, P.E. Sacks, and A.V. Tikhonravov, Inverse Problems 11 (1995) 
[16] G. Reiss, this conference.

[17] E.J. Kramer. Physica B 173 (1991) 189.

[18] T.L. Crowley, E.M. Lee, E.A. Simister, and R.K. Thomas, Physica B 173 (1991) 143.

[19] E.M. Lee, this conference.

[20] D.S. Sivia and R. Pynn, SPIE 1738 (1992) 305.

[21] C.F. Majkrzak, N.F. Berk, J.F. Ankner, S.K. Satija, and T.P. Russell, SPIE 1738 (1992) 282.

[22] V.O. de Haan, A.A. van Well, S. Adenwalla, and G.P. Felcher, Phys. Rev. B (in press).

[23] C.F. Majkrzak and N.F. Berk, Phys. Rev, B (in press) and this conference.

[24] see e.g. K. Chadan and P. Sabatier, 'Inverse Problems in Quantum Scattering Theory', (Springer Verlag, Berlin, 1989).

[25] D.S. Sivia, W.A. Hamilton, and G.S. Smith, Physica B 173 (1991) 121.

[26] V.S. Buslaev and V.N. Fomin, Vestnik Leningrad Univ. 17 (1962) 56.

[27] A. Cohen and T. Kappeler, Indiana Univ. Math. J. 34 (1985) 127.

\section{Figure captions}

Fig. 1 Scattering-length density profiles $\Gamma(z)$ as a function of the depth $z$, for a 25 nm thick gold layer. Au00: sharp interfaces; Au01: sharp first and rough second interface; Au10: rough first and sharp second interface; Aul1: both interfaces rough. The $\mathrm{rms}$ roughness parameter $\sigma=2.5 \mathrm{~nm}$.

Fig. 2 Amplitude and phase of the complex reflection coefficient $r(q)$, for the four systems, displayed in Fig. 1. Au00: solid line; Au01: dash-dotted line; Au10: dashed line; Aull: dotted line. Note that $\operatorname{Ir}(q) !$ of Au01 is shifted by a factor 0.8 , and $\arg (r(q))$ of Au11 shifted by a constant equal to 1 .

Fig. 3 Representation of the reflection coefficient $r(q)$ in the complex plane for the four scartering-length density profiles, shown in Fig. 1. For the systems Au00, Au01, Au10, and Aul 1, $q^{2} r(q)$ in the $q$ range from 0 to $0.5 \mathrm{~nm}^{-1}$ is displayed. Note that $r(q=0)=-1$. 
Fig. 4 System used in 'computer experiment'. (a) Experimental geometry, with cobalt as tunable reference layer and a gold layer as 'unknown' layer. Both the layer with sharp first and rough second interface (Au01) and vice versa (Aul0) are investigated. (b) Scattering-length density profile for the three 'measurements': polarized beam 'spin-up' $(+)$ : dash-dotted line; and 'spindown' (-): solid line; and unpolarized beam with Co magnetized along the $z-$ axis $(\perp)$ : dashed line.

Fig. 5 (a) Three simulated measurements for system Co/Au01, with $5 \%$ statistics added. (b) amplitude and (c) phase of the retrieved reflection coefficient of the gold layer (Au01). 'Perfect data' (no statistics added): continuous line; 'noisy data' (5\% statistics added): pluses with error bars. Note that the retrieved $r(q)$ in the case of 'perfect data' are identical to results, calculated with the matrix method (Fig. 2).

Fig. 6 As Fig. 5, for system Au10.

Fig. 7 Three circles in the complex plane, each representing one of the three 'measurements' (Eqs. (6-7)), for three different values of q. ( + ): dash-dotted line; $(\perp)$ : dashed line; $(-)$ : solid line. The plus sign indicates the intersection point calculated according to Eq. (15). (a) System Au01, 'perfect data'. (b) System Au01, 'noisy data'.

Fig. 8 Scattering-length density as a function of depth $z$ for systems (a) Au01 and (b) Au10: Original profile: line. Recovered profiles: 'perfect data': open circles; 'noisy data': triangles; 'mixed data': filled circles $\left(q<0.076 \mathrm{~nm}^{-1}\right.$ : perfect $r(q)$ data, $q>0.076 \mathrm{~nm}^{-1}$ : noisy $r(q)$ data). 
Fig.1
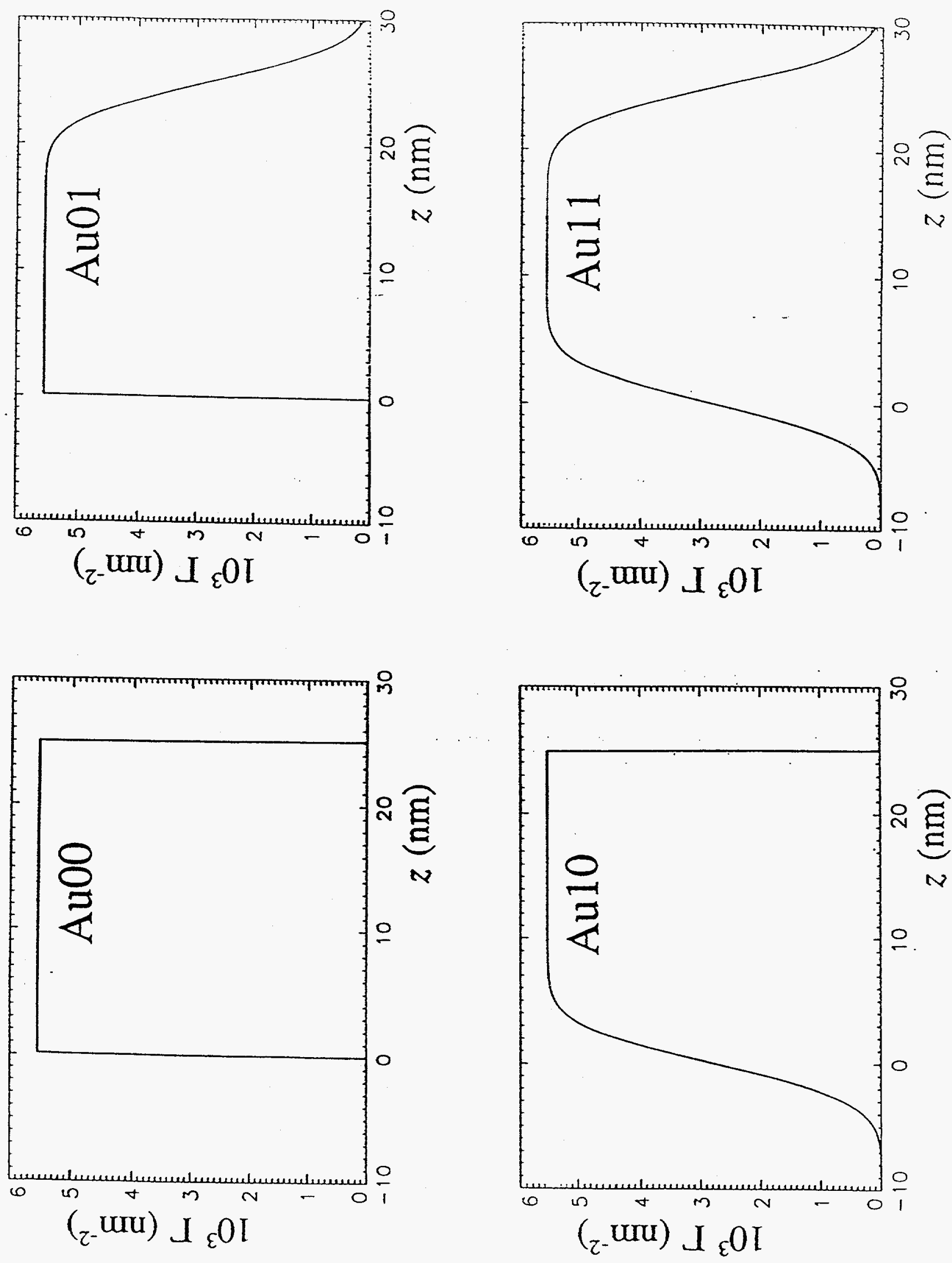

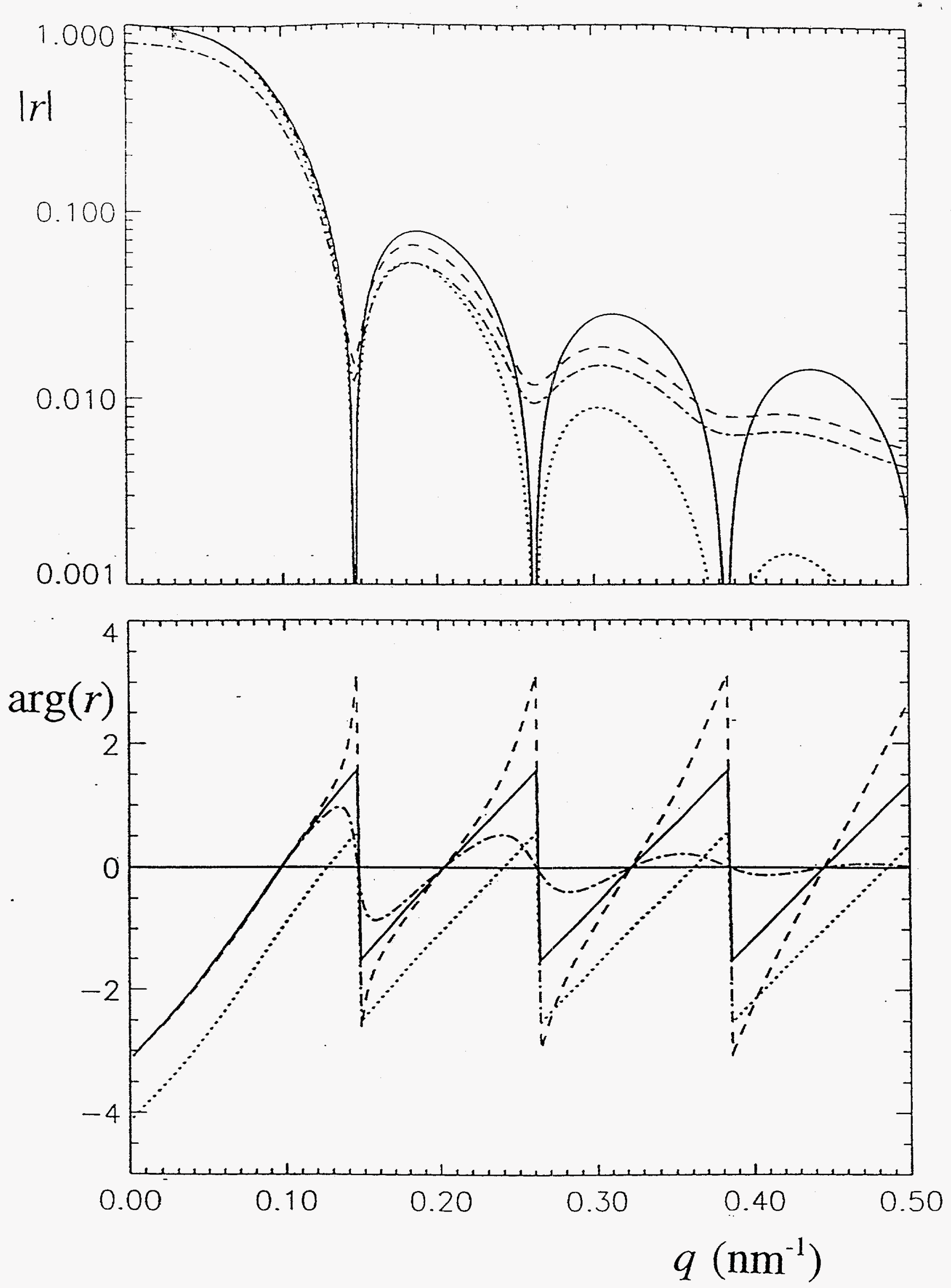

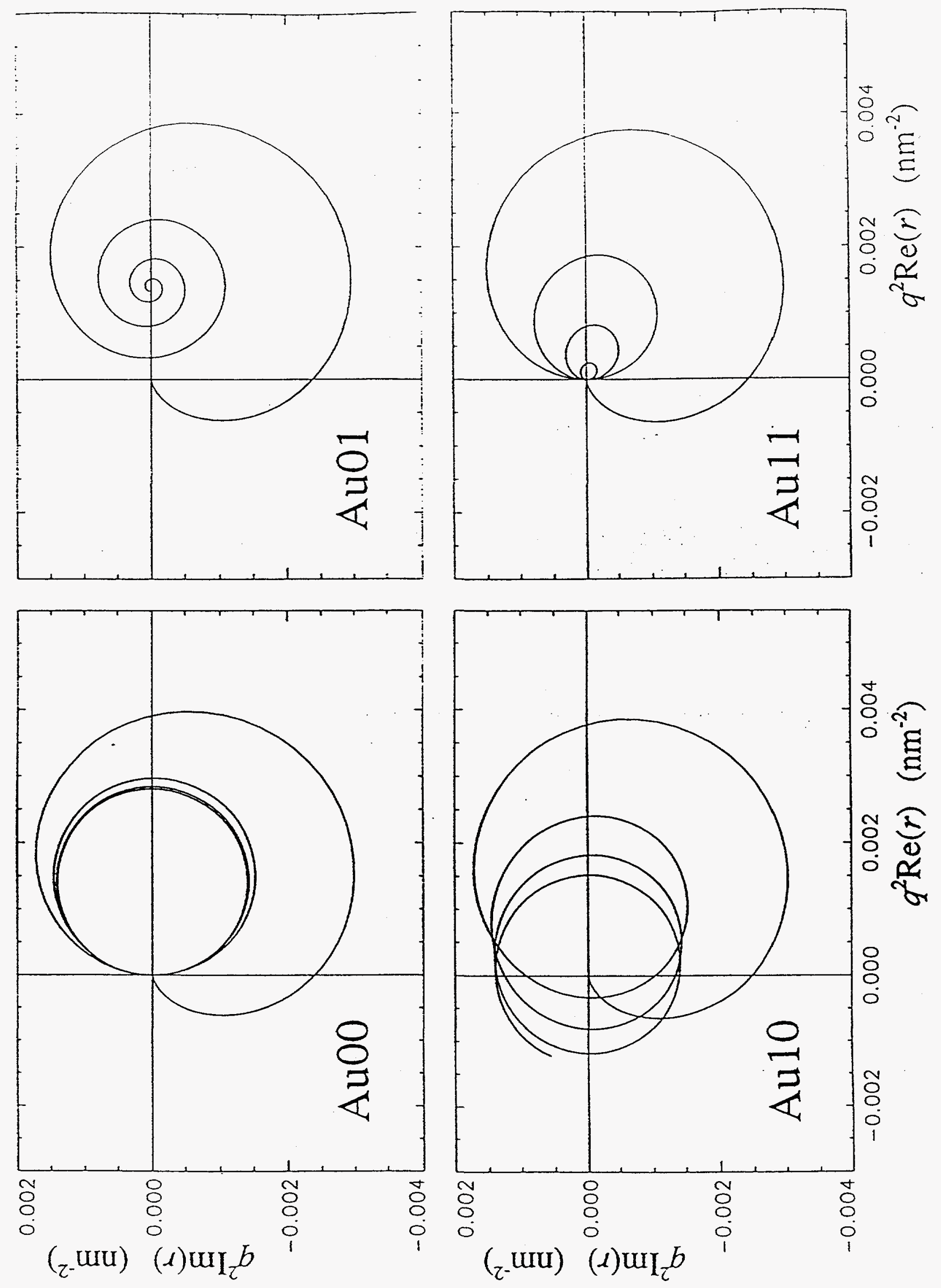


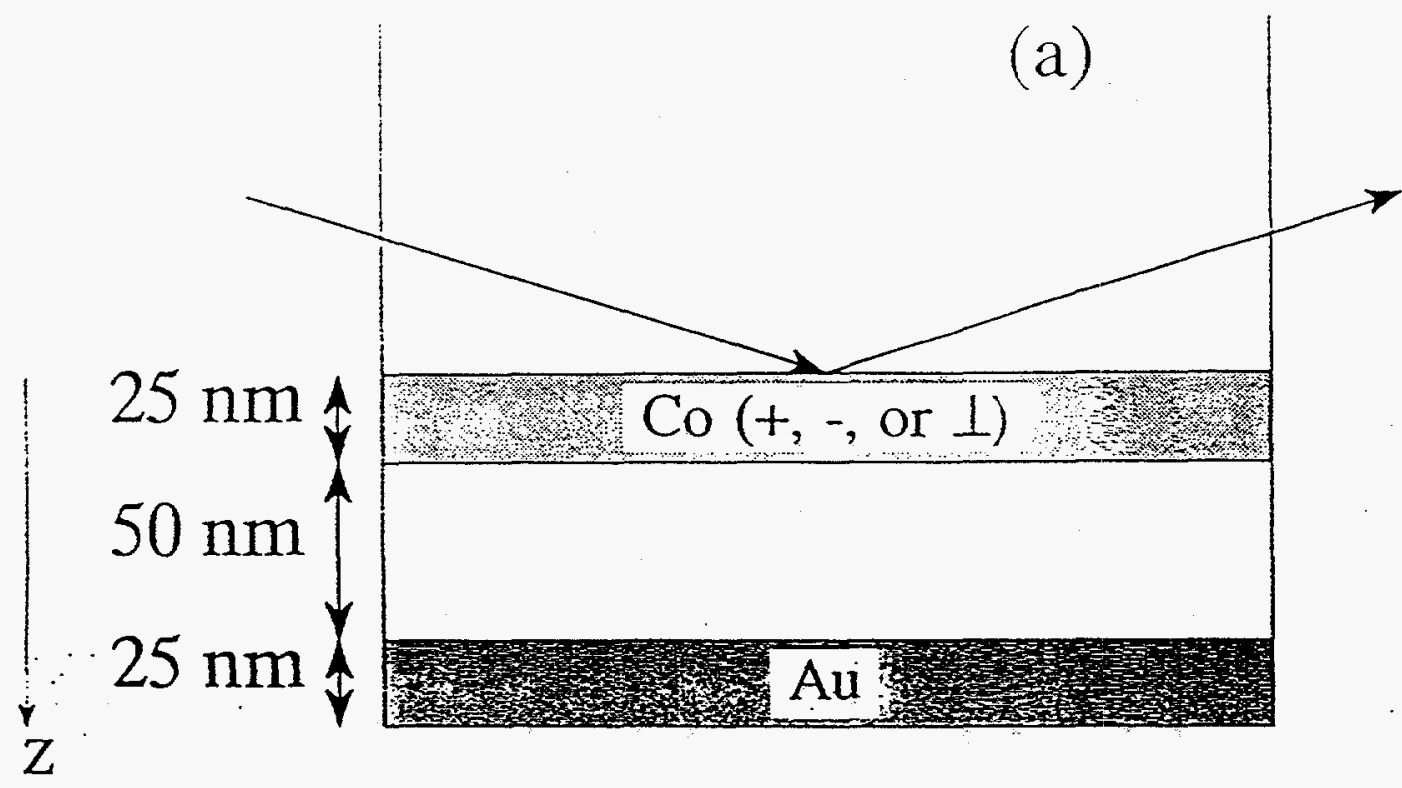

'known'

'unknown'

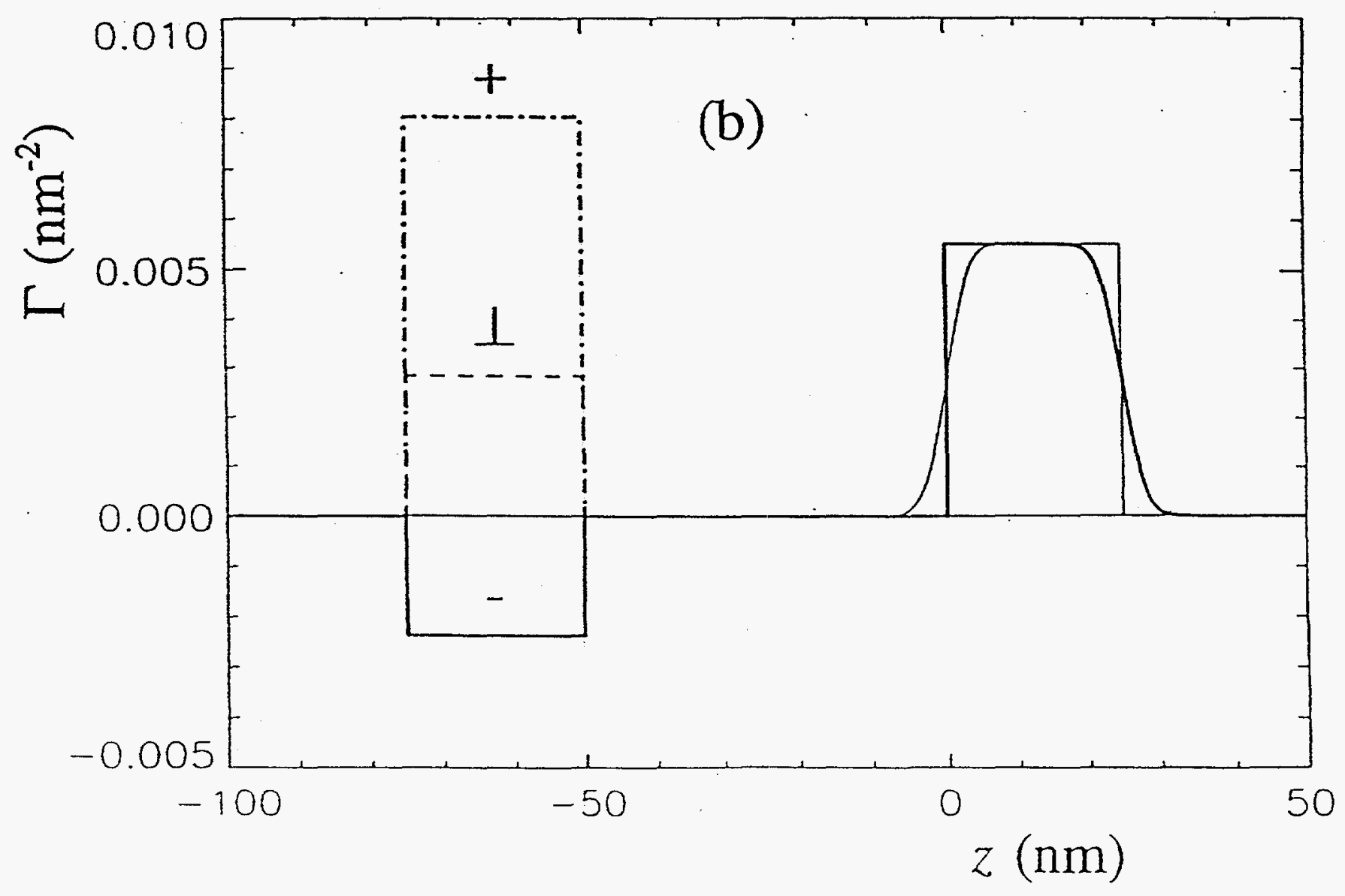



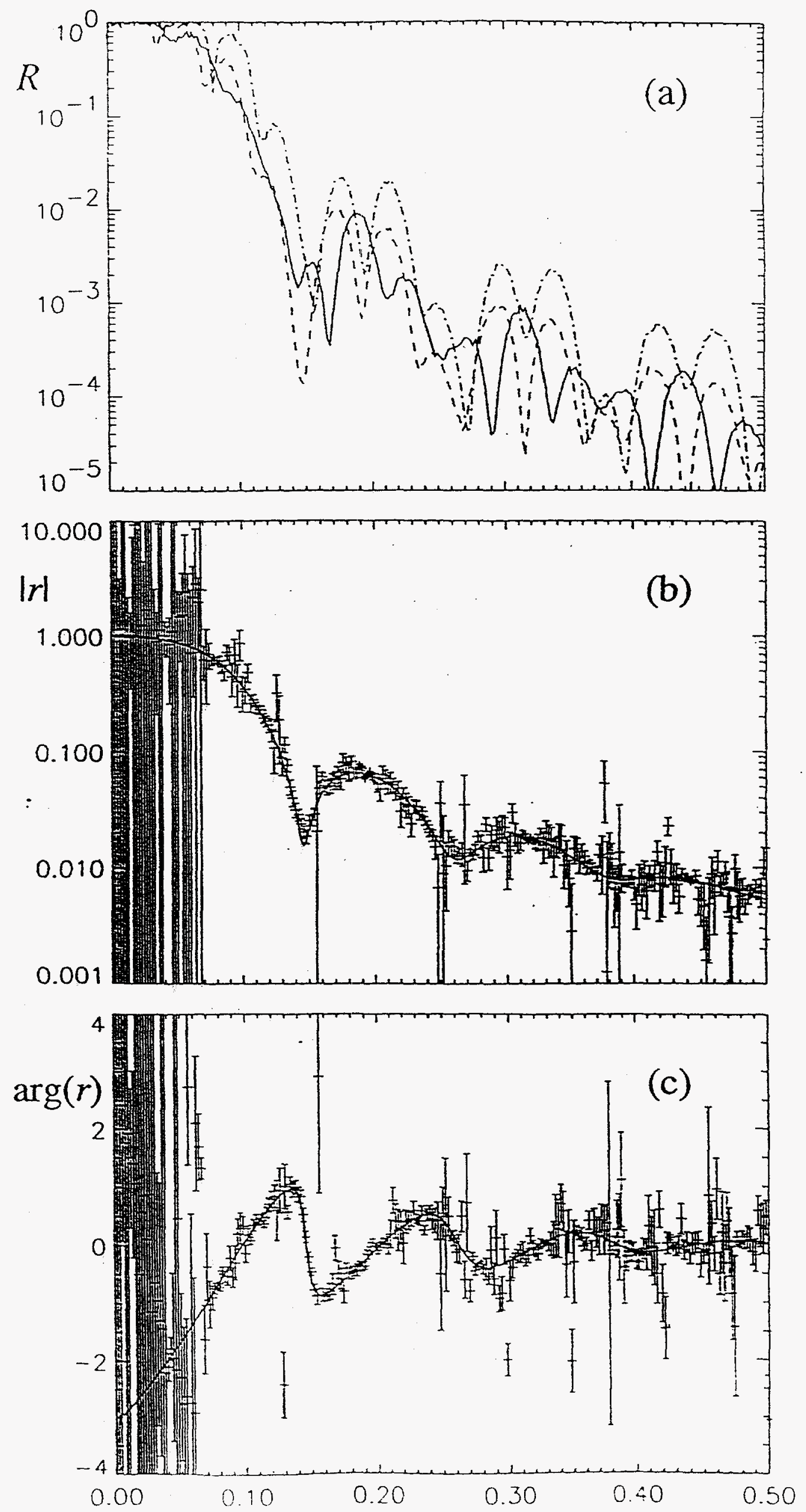